\title{
CONTINUOUS INTRAPARTUM FETAL SCALP TISSUE $p H$ AND ECG MONITORING BY A FIBEROPTIC PROBE
}

Howard M. Hochberg, M.D.

Paul V. Roby, M.D.

Hattie M. Snell, R.N.

W. Dean Smith, B.S.

International Biomedics, Inc., Bothell, Washington 98021

Molly S. Chatterjee, M.D.

Our Lady of Mercy Medical Center, Bronx, N.Y. 10466

\section{Introduction}

Labor and delivery can rapidly change from a normal to a potentially high risk situation. Accordingly, careful monitoring of mother and fetus has become the standard of care. Continuous electronic "fetal" monitoring (EFM) of fetal heart rates and uterine contractions is used almost universally in the United States. EFM information is sensitive to the possibility of dangerous deficits in fetal oxygen supply. However EFM data yields about 50 to $60 \%$ false positive "diagnoses" of significant fetal hypoxia (1) and may not detect up to $15-20 \%$ of true hypoxia. The most specific index of prolonged and potentially dangerous insufficient oxygen delivery is fetal blood $\mathrm{pH}(\mathrm{BpH})$; measured from drops of blood carefully collected from a small incision on the fetal scalp. The combination of BpH and EFM data reduces the incidence of false positives, while also improving detection of true problems. These combined data can be used to substantially reduce the Cesarean section rate for fetal distress (1-4). It is estimated that $\mathrm{BpH}$ information may be clinically important in up to $25 \%$ of patients $(3,5)$.

However, $\mathrm{BpH}$ data is not commonly available because of the skills, time, inconvenience and laboratory facilities needed to obtain reliable measurements. Further one $\mathrm{BpH}$ measure is insufficient to 
determine the trend of fetal $\mathrm{pH}$ (5). Finally, the tools and methods are primitive and there is some fetal risk (2). For all these reasons, and despite its potential value, $\mathrm{BpH}$ has not become the standard of practice in the United States.

The information inherent in the $\mathrm{BpH}$ measurement could be made widely available if there were a convenient safe probe to continuously monitor intrapartum fetal $\mathrm{pH}$. This paper describes an investigational fetal $\mathrm{pH}$ probe designed to be safe and convenient. At this time, the goal of the device is to provide reliable fetal pH status and trend data for use as an adjunct to EFM and BpH data for clinical care.

\section{Historical Perspective}

The first fetal $\mathrm{pH}$ probe to undergo widespread clinical trials was the Stamm tissue $\mathrm{pH}(\mathrm{tpH})$ probe, designed for monitoring the $\mathrm{pH}$ of the subcutaneous fluid in the fetal scalp $(6,7)$. Several studies demonstrated the validity of the basic concept of Stamm: human fetal acid-base balance can be monitored and evaluated by continuous monitoring of the scalp tpH (8-12). Clinical utility included a reduction in operative intervention for deliveries (9). However the Stamm device required sterile assembly and charging with sterile reference electrolyte a scalp incision for probe insertion and mastery of a difficult technique for apply and fixing the probe to the fetal scalp. The system was found to be impractical and the technique unreliable for routine clinical use.

In order to help to make these potentially valuable fetal tpH data generally available the authors and clinician investigators are evaluating a system and tpH probe designed to be convenient, safe, and reliable for continuous monitoring of fetal $\mathrm{pH}$ status. Fiberoptic, electro-optic, microprocessor and analytic chemistry technologies were integrated to produce a feasibility system designed for evaluation of continuous clinical monitoring of the fetal $\mathrm{pH}$ status during labor: The $\mathrm{OBpH}^{\mathrm{TM}} 1000$ System. Using the experience gained 
in monitoring over 300 patients between late 1983 through mid 1985, a total system designed to be convenient and simple for routine use by the clinician was designed and built. The latter system, the OBpH 1500, is in evaluation at this time. This paper describes the $\mathrm{OBpH} 1500$ system and outlines clinical results from the earlier OBpH 1000 system, which used the same tpH probe.

Because this is an unreleased FDA Class III Investigational Devise in evaluation for regulatory purposes, International Biomedics can make no claims regarding safety, effectiveness or utility of the system. Further, it should be understood the device is designed as an adjunct to EFM and fetal scalp blood sampling, not as a total replacement for either measurement. (1987)

\section{System Description}

The OBpH 1500 System includes a fetal scalp pH ECG probe, an applicator tool, an electro-optic Interface module, an off-line Calibration chamber, and a fetal monitor for data display (Figures 1,2). The probe is a 22 gauge spiral ECG electrode which includes a fiberoptic-based pH sensor (Fig. 3,4). The probe is applied to the fetal scalp with the same general technique used for spiral ECG electrodes. When properly placed within the scalp, the $\mathrm{pH}$ sensor is within the subcutaneous fluid and the probe detects both subcutaneous fluid (tissue) $\mathrm{pH}$ and fetal ECG.

The probe is supplied in a sterile pack, with the tpH sensor within $\mathrm{pH} 7.00$ buffer. After insertion of the probe pack and connector into the Interface, and insertion of the Interface into the Calibrator, the probe is automatically warmed, stabilized, stored and calibrated within the sterile pack. PH 7.36 Calibration is begun by depressing a sealed plunger through the cover of the sterile probe pack. The Calibrator checks for problems, confirms the reliability of the calibration, and the probe is ready for use within about 15 minutes. The calibrated probe can be used with confidence for up to 8 hours. 


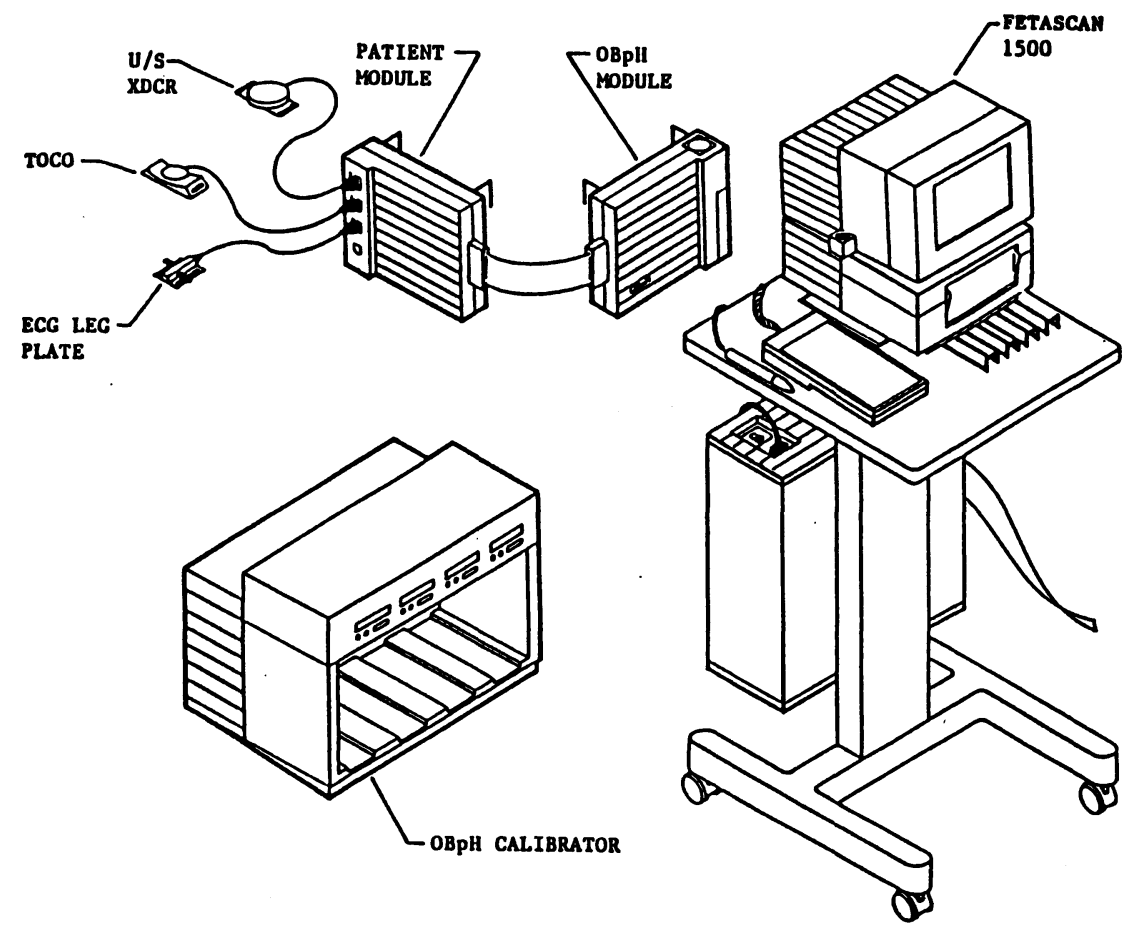

Figure 1 The OBpH 1500 System

This illustrates the bedside monitoring configuration of the system. The $\mathrm{OBpH}$ Module (Interface), an electro-optic and memory module which can contain the $\mathrm{OBpH}$ probe set. The $\mathrm{OBpH}$ Interface mates with the Patient Module of the FetaScan 1500. This assembly can be placed at the bedside, as the Patient Module accepts the ECG leg plate cable and uterine contraction transducers and the $\mathrm{OBpH}$ Interface can include a calibrated probe ready for use.

The $\mathrm{OBpH}$ Interface acquires and retains the probe calibration data, allowing the patient to be disconnected from the monitor for transport by disconnecting the Interface from the Patient Module. 


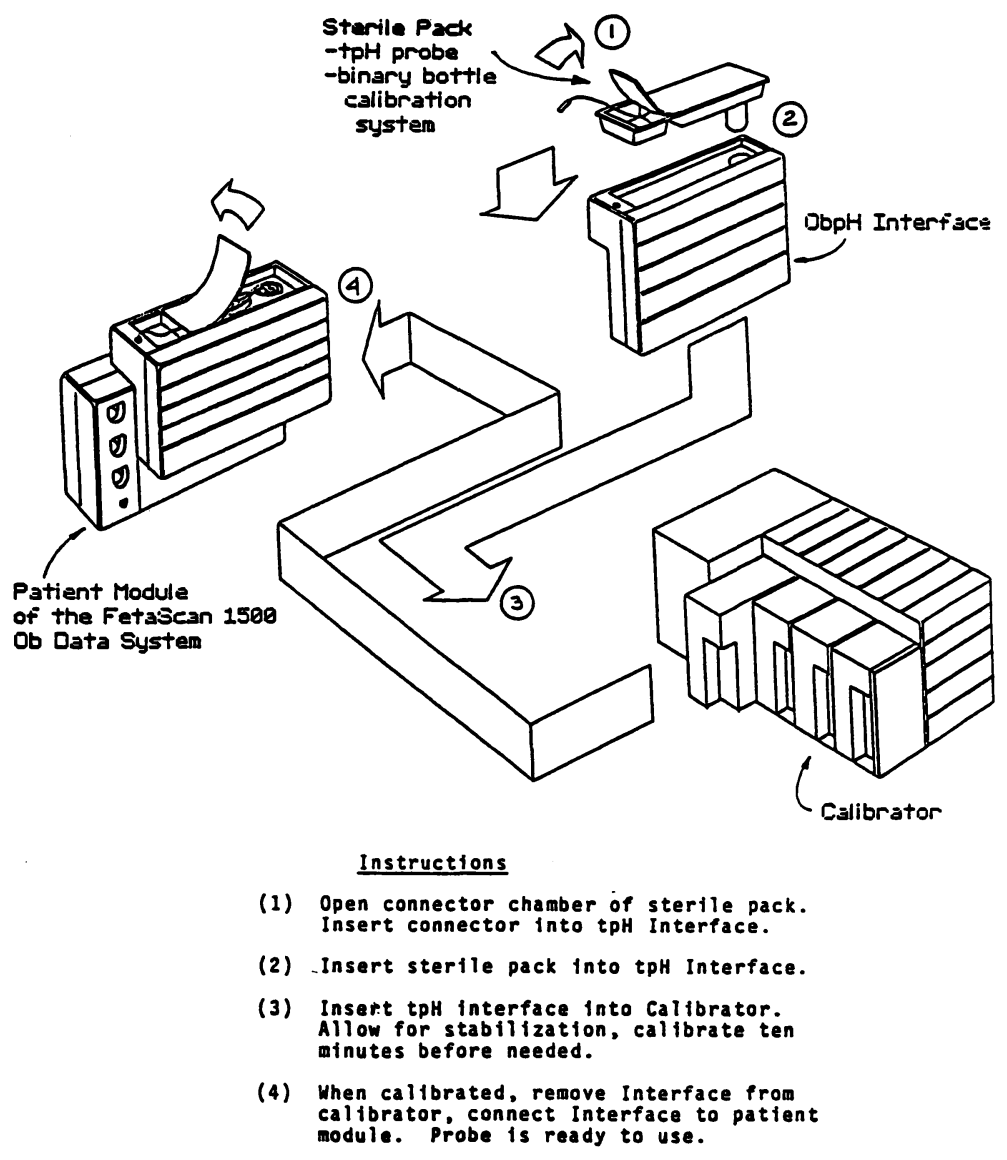

Figure 2 Preparation of the Model $1500 \mathrm{OBpH}$ System

The $\mathrm{OBpH} 1500$ Interface mates with either the calibrator or the monitor. It acquires and retains the probe calibration data, allowing the patient to be disconnected from and reconnected to any appropriate FetaScan 1500 monitor by disconnecting and reconnecting the interface, without disconnecting the probe.

The $\mathrm{OBpH}$ probe is in a sterile sealed package and it can be fully calibrated in this package without impairing sterility. Once calibrated, it can be placed at the bedside ready for use. The semiautomatic Calibrator holds 4 probe-interface packages ready for final calibration and use. 


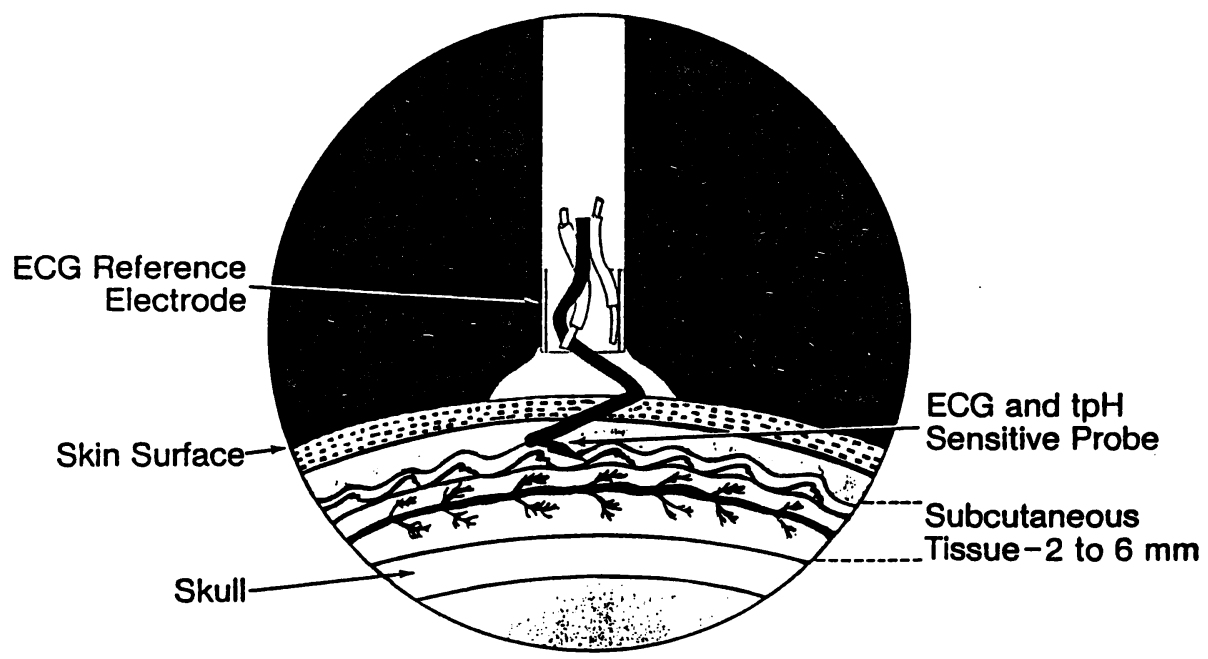

Figure 3

Drawing of the tpH probe in situ in the fetal scalp, with the applicator tool attached. The spiral forms one pole of the ECG electrode and the hub is the other. The tpH sensor window is just below the skin surface, facing into the subcutaneous tissue in order to measure the $\mathrm{pH}$ of the interstitial fluid. 


\section{Fiberoptic pH Probe Design}

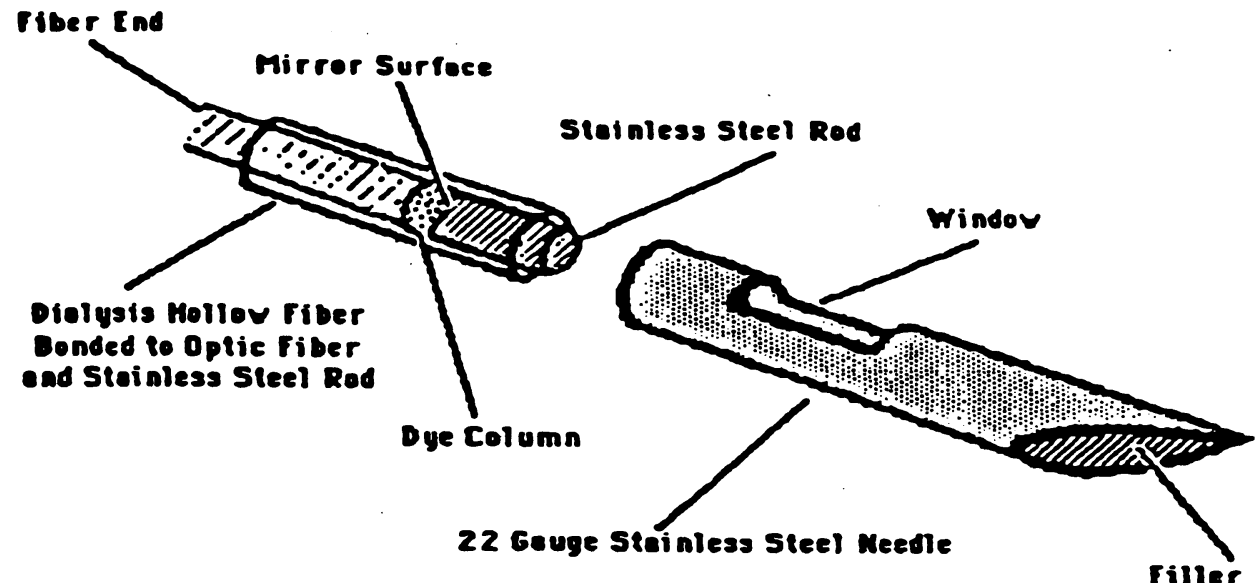

Figure 4 Principle of Operation of the Fiberoptic pH Probe

The above assembly is inserted into the solution to be measured. Protons $(\mathrm{H}+)$ diffuse freely through the dialysis hollow fiber into the dye column. The column contains a reversible $\mathrm{pH}$-sensitive non-toxic dye covalently bound to a gel matrix (to prevent escape of dye). A fiberoptic fiber guides light to the dye, and directs reflected light to the instrument. There the green reflectance, the red reflectance, and the current with no light (dark current) are measured. The absorbance of green light by the dye is proportional to the $\mathrm{pH}$ in the range of interest, while red absorbance is not affected by $\mathrm{pH}$. The red relectance is used as a reference signal. 
The Interface module, with probe still attached, is then moved to the bedside. The pack is opened and the probe applied to the fetal scalp. The interface module is then attached to the FetaScan monitor, which automatically acquires and merges the $\mathrm{tpH}$ with the monitoring data on its strip chart and the video display, and continuously checks the validity of the optical data in order to flag problems (Fig. 5).

\section{INTRAPARTUM FETAL tPH MONITORING}

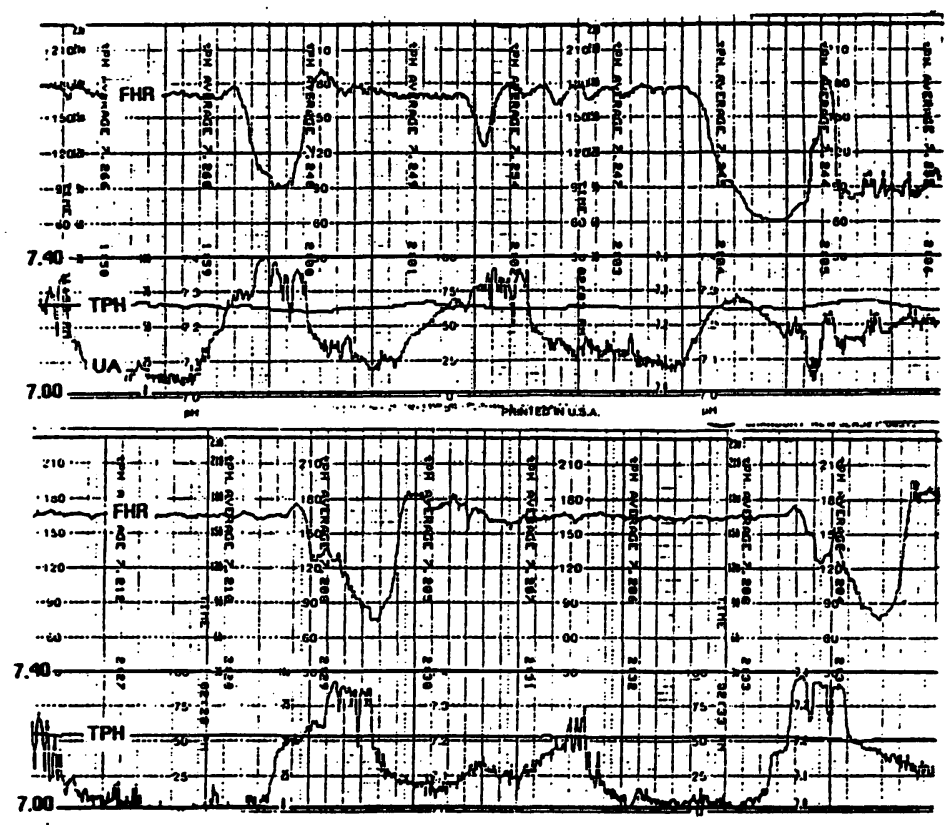

\section{Figure 5 Chart Display of Simultaneous Fetal Monitor and OBpH Data}

The fetal heart rate (FHR) demonstrates significant decelerations concordant with uterine activity (UA). The UA channel includes a tpH scale which ranges from $\mathrm{pH} 7.00$ (bottom of record) to 7.40 (top of UA channel). The tpH is displayed as a linear graph on the UA channel, and it is updated every 20 seconds with the average tpH for the past 20 seconds. Each minute the average tpH is printed on the FHR channel (vertical printed statements). The FetaScan video displays the 20 second updated tpH value. 


\section{In-Vitro Performance of the $\mathrm{OBpH}$ System}

In order to be useful as a trend monitor the $\mathrm{OBpH}$ system must resolve changes in $\mathrm{pH}$ at least as well as the current technology: the $\mathrm{BpH}$. The $95 \%$ confidence interval (ci) of the $\mathrm{BpH}$ measurement is reported to be $0.06 \mathrm{pH}$ or greater, with a non-Gaussian distribution $(13,14)$. In-vitro studies of the $\mathrm{OBpH}$ probe demonstrate the $95 \% \mathrm{ci}$ is $0.03 \mathrm{pH}$ (Fig. 6). The absolute accuracy is within $\pm 0.05 \mathrm{pH}$ of a blood gas analyzer, which is also within the $95 \%$ confidence limits of blood measurement. Published data $(8,15)$ show the high degree of correlation $R>0.9$ with in-vitro measurements of human fetal and adult blood.

\section{Prow 879}

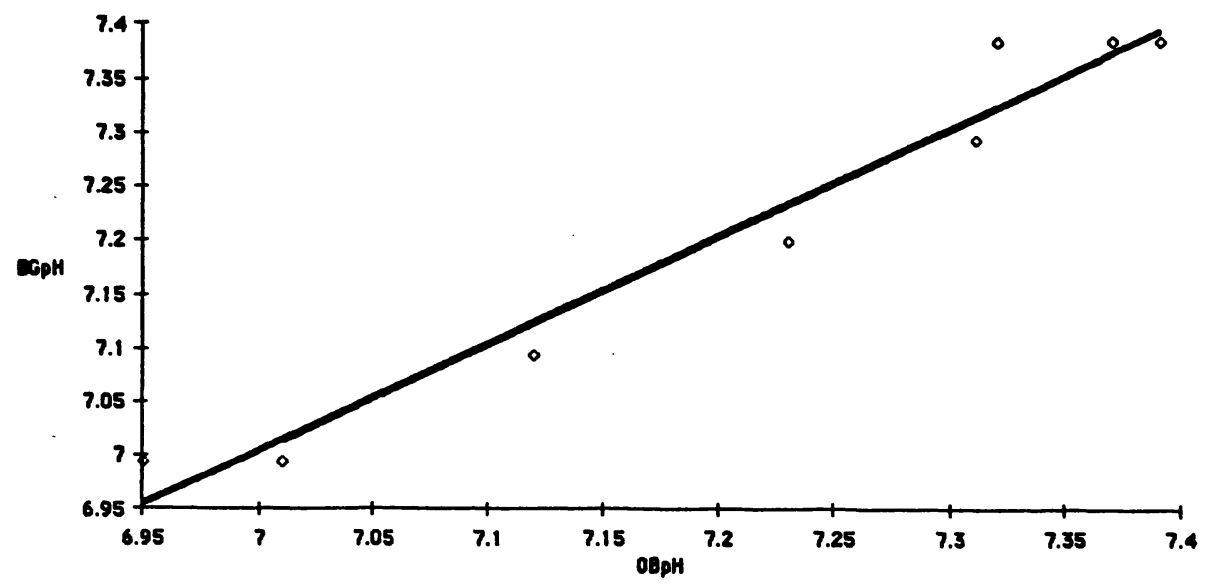

\section{Figure 6 Reproducibility of $\mathrm{pH}$ Measurement with a Single} Fiberoptic tpH Probe

The $\mathrm{OBpH} 1500$ data is compared to blood gas analyzer $\mathrm{pH}(\mathrm{BGpH})$ at eight data points. The probe is first calibrated and then immersed in buffers of six other $\mathrm{pH}$, and it is recycled through all eight $\mathrm{pH}$ at least twice. The probe must reproduce each data point within a bandwidth of $\pm 0.03 \mathrm{pH}$. 


\section{Clinical Investigational Plan}

The feasibility model $1000 \mathrm{OBpH}$ system was used in eight U.S. centers to monitor over 300 term or post-date high risk patients where $\mathrm{BpH}$ was indicated. The $\mathrm{BpH}$ was used to document the reliability of the current technology, the $\mathrm{BpH}$, in following trends and to validate the reliability and the $\mathrm{OBpH}$ monitor in following trends of fetal $\mathrm{pH}$ status and in classifying fetal $\mathrm{pH}$ status as normal vs. notnormal.

The study goals:

1. Document the incidence of fetal or maternal problems caused by the $\mathrm{OBpH}$ in order to compare its safety with current technology.

2. Measure the inherent reliability of the tpH data for monitoring fetal $\mathrm{pH}$ status. "Inherent" means both the $\mathrm{BpH}$ and the $\mathrm{OBpH}$ data were unflawed or untainted, according to the investigator's report and an independent non-clinician reviewer at International Biomedics.

3. Document the perceived added value and clinical relevance of the $\mathrm{OBpH}$ monitoring data, when used as an adjunct to EFM and $\mathrm{BpH}$ measurements.

\section{Clinical_Study Methods}

- Subject selection criteria:

- Term or post-date patients in labor with vertex fetal presentation, and sufficient dilation and station to properly place the probe.

- A documented indication for fetal scalp blood sampling for $\mathrm{pH}$ measurements.

- No contraindications to fetal scalp blood sampling for $\mathrm{pH}$ measurements.

- Informed consent given. 
- Study Population: (Table I)

TABLE I

Study Population

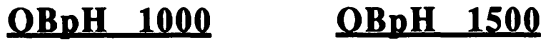

Total Patients

296

30

Total Formal Reports

291

30

Average Maternal Age

25 years

25 years

Average Gestational Age

39.9 wks

40.5 wks

Average Duration of $\mathrm{OBpH}$

Monitoring

2.0 hours

2:23 hours

Indications for $\mathrm{pH}$ Monitoring (\% Cases)

$\begin{array}{lrc}\text { Abnormal FHR } & 83 \% & 39 \% \\ \text { Meconium } & 32 \% & 28 \% \\ \text { Post-date } & 21 \% & 40 \% \\ \text { Hypertension } & 16 \% & 4 \% ? \\ \text { Diabetes } & 6 \% & -- \\ \text { Other } & 46 \% & 36 \%\end{array}$

Pertinent Medications (\% Cases)

$\begin{array}{lrl}\text { Oxytocin } & 42 \% & 27 \% \\ \text { Magnesium Sulfate } & 12 \% & -0- \\ \text { Tocolytics } & 3 \% & -0-\end{array}$

Mode of Delivery (\% Cases)

Spontaneous Vaginal

$61 \%$

$83 \%$

Cesarean section

$26 \%$

$17 \%$

Forceps

$11 \%$

$-0-$

Vacuum

$1 \%$

No data

$1 \%$

-0 -

-0 - 
TABLE I (CON)

Study Population

$\underline{\mathrm{OBpH} 1000} \quad \underline{\mathrm{OBpH} 1500}$

Neonatal Problems

Nuchal cord

Respiratory difficulties

$9 \%$

$10 \%$

Meconium aspiration

$3 \%$

$3 \%$

Other

$3 \%$

-0 -

$7 \%$

$-0-$

One Minute Apgar Scores

$-3$

$6 \%$

$-0-$

4- 6

$18 \%$

-0 -

$7-10$

$78 \%$

$100 \%$

Adverse Effects

$3.8 \%$

-0 -

- "Qualified Data Analysis" was used to measure the intrinsic reliability of the $\mathrm{OBpH}$ to monitor (correlate with) fetal $\mathrm{CpH}$ measurements, given no experimental flaws or "tainted data" and given the inherent variability of the $\mathrm{CpH}$ data. The correlation of replicate fetal $\mathrm{CpH}$ measures was determined in order to quantify the reliability of the current technology. Next, the tpH-CpH correlation data were calculated for those data points with replicate $\mathrm{CpH}$, and then for all qualified sets of related tpH-CpH (Table II).

- "Physiological Data Analysis" was used to measure the clinical reliability of $\mathrm{OBpH}$ in trending fetal $\mathrm{pH}$ status, as measured by the correlation coefficient, given no obvious problems known to the investigator and $\mathrm{pH}$ values in the system operating range 7.09-7.44. (Tables II \& III).

- Reliability of classification of fetal $\mathrm{CpH}$ by the $\mathrm{OBpH}$ data. These data included the predictive value of a normal tpH (7.20-7.44) vs. a normal $\mathrm{CpH}(7.25+)$ and the accuracy of both normal and not normal tpH to respectively predict normal \& not normal $\mathrm{CpH}$ (efficiency of 
tpH). These data quantified the reliance the clinician can place as the tpH reflecting the $\mathrm{CpH}$ status. (Tables II \& III).

- Success in obtaining a reliable $\mathrm{OBpH}$ trend record as compared to the success of obtaining reliable $\mathrm{CpH}$ measurements was measured. Certain conditions are known to interfere with blood $\mathrm{pH}$ and fetal scalp ECG reliability because free access to the scalp is blocked, and these similarly interfere with $\mathrm{OBpH}$ probe fixation. The most notable in this study was thick matted fetal hair. Meconium, scalp edema and duration of monitoring or labor did not consistently interfere with $\mathrm{OBpH}$ or $\mathrm{BpH}$ data.

- The potential clinical relevance of the $\mathrm{OBpH}$ data in patient management in addition to the FHR and $\mathrm{BpH}$ data was reported on the data forms and tallied.

TABLE II

Reliability of the $\mathrm{OBpH} 1000$ Data (Feasibility Model)

Total Case Reports with

$\mathrm{CpH}$ and $\mathrm{OBpH}$ Data

198

Correlation Coefficients

Weighted Mean Range

(among Institutions)

\section{Qualified Data}

BpH/BpH: Blood $\mathrm{pH}$ reliability: $\quad \mathrm{r}=0.81 \quad \mathrm{r}=0.71-0.90$

(excluding Corometrics 220

Analyzer $\mathrm{CpH}$ data)

$\mathrm{OBpH} / \mathrm{BpH}$ (Trending reliability): $\quad \mathrm{r}=0.84 \quad \mathrm{r}=0.72-0.94$

Reliability of Classification of $\mathrm{BpH}$ by $\mathrm{OBpH} 1000$ Data

Unflawed Physiological Data: $\quad n=213$ pairs.

Predictive Value of Normal tpH: $100 \%$

False normal tpH: $\quad 0 \%$

Efficiency (correct class./total): $\quad 68 \%$ 
TABLE III

Reliability of the $\mathrm{OBpH} 1500$ Data

Total Case Reports with both $\mathrm{CpH}$ and $\mathrm{OBpH}$ Data

20 (2 institutions)

\section{Correlation Coefficients}

Weighted Mean $\underline{\text { Range }}$

Physiological Data

$\mathrm{BpH} / \mathrm{BpH}$ : Blood $\mathrm{pH}$ reliability: Insufficient Data

$\mathrm{OBpH} / \mathrm{BpH}: \quad r=0.89 \quad r=0.79-0.93$

\section{Reliability of Classification of $\mathrm{BpH}$ by $\mathrm{OBpH} 1500$ Data}

$\begin{array}{ll}\text { Unflawed Physiological Data: } & \mathrm{n}=20 \text { pairs } \\ \text { Predictive Value of Normal tpH: } & 100 \% \\ \text { False Normal OBpH: } & -0- \\ \text { Efficiency (correct class/total): } & 89 \%\end{array}$

\section{Success in Obtaining Fetal pH Data}

The clinical experience demonstrated a continuous $\mathrm{OBpH}$ monitoring record could be obtained as frequently as a single valid fetal scalp blood $\mathrm{pH}$ data point $(80 \%)$. With the $\mathrm{OBpH} 1500$, the process was perceived to be simpler than obtaining one set of fetal scalp blood $\mathrm{pH}$ data point.

\section{Clinical Relevance of the OBpH Data}

The OBpH data was perceived as clinically relevant in $65 \%$ of these case reports. The most commonly mentioned advantages were:

-Reassurance of fetal pH stability and normalcy, especially despite other changes suggestive of possible fetal deterioration. (Abnormal FHR, Thick Meconium) This impression was validated by the $100 \%$ predictive value of a normal tpH. 
-Avoiding extra fetal scalp blood samples.

-Detecting trends in pH before they can be resolved by the blood pH method. (Because of the $\mathrm{OBpH}$ self consistency)

-Using the $\mathrm{OBpH}$ record as a guide to timing fetal scalp sampling for blood pH. Usually there is no clear-cut indication as to when the pH is changing and may need diagnostic confirmation. The $\mathrm{OBpH}$ trend uniquely provides this information.

-Monitoring fetal pH status where it is impractical to obtain timely repetitive blood $\mathbf{p H}$ data. This is particularly relevant during stage II of labor, when abnormal heart rate patterns are common.

\section{Discussion and Conclusions}

The data demonstrated the $\mathrm{OBpH}$ system and the tpH method was inherently capable of reliable monitoring of fetal $\mathrm{pH}$ status, albeit with a different absolute value. The inherent consistency of the $\mathrm{OBpH}$ was obscured by the variability of the current technology, the $\mathrm{BpH}$, the additional inter-institutional difference in $\mathrm{BpH}$ reliability; and possibly by clinical factors such as thick fetal hair. Also, there is an initial learning curve effect. At the start of the program, the correlation coefficient of tpH to $\mathrm{OBpH}$ was about 0.6. The last studies revealed correlation coefficients of 0.8 to 0.95 with a few or no tainted data points. Throughout the study, there were no false normal tpH values. When the tpH was 7.25 to 7.44 , the unflawed $\mathrm{BpH}$ was always in the normal range.

Most clinicians perceived the data from the $\mathrm{OBpH}$ system to be clinically relevant, especially in monitoring trends of fetal $\mathrm{pH}$ status during worrisome events or events where repetitive timely $\mathrm{BpH}$ cannot be obtained. The investigators also immediately perceived the reliability of a normal and stable $\mathrm{OBpH}$ record.

To date, the clinical results obtained from the $\mathrm{OBpH} 1500$ system are encouraging with respect to both practicality and reliability of the data. 


\section{References}

(1) Anonymous: Antenatal Diagnosis. NIH Concensus Development Conference. March 5-7, 1979. US Dept HEW PHS NIH. NIH Publication No 79 (1973)

(2) Young DC, JH Gray, ER Luther et al: Fetal scalp blood pH sampling: Its value in an active obstetric unit. Am J Obstet Gynecol 126 (1980) 276

(3) Van den Berg P, S Schmidt, J Gesche, E Saling: Fetal distress and the condition of the newborn using cardiotocography and fetal blood analysis during labour. Brit J Obstet Gynaecol 94 (1987) 72

(4) Lagercrantz H, TA Soltkin: The "stress" of being born. Scientific American 2544 (Apr. 1986) 100

(5) ACOG: ACOG Technical Bulletin 42 (1976): Fetal blood sampling.

(6) Stamm O, U Latscha, P Janacek, A Campana: Kontinuierliche $\mathrm{pH}$-Messung am kindlichen Kopf post partum und sub partu und post partum (Continuous subcutaneaous assy of $\mathrm{pH}$ on the head of the infant after and during delivery). Z Geburtshilfe Perinatol. 178 (1974) 368

(7) Stamm O, P Janacek, A Campana: Registro continuo del pH tissular en perinatologia. Acta Ginecologica 26 (1975) 165

(8) Chatterjee M, F Hetzel, A Kaminetzky: Fetal tissue pH - Continuous intrapartum monitoring. Int J Gynaecol Obstet 22 (1984) 41

(9) Weber $\mathrm{T}: \mathrm{pH}$ monitoring during labour with special reference to continuous fetal scalp tissue pH. Danish Medical Bulletin 30 (1983) 215

(10) Lauersen NH, FC Miller, RH Paul: Evaluation of continuous fetal scalp $\mathrm{pH}$ during labor. Arch Gynecol 226 (1978) 141

(11) Kellner KR, TC Key, AC Cruz, WN Spellacy: Evaluation of a continuous tissue $\mathrm{pH}$ monitor in the human fetus during labor. Obstet Gynecol 55 (1980) 523

(12) Flynn AM, J Kelly: The continuous measurement of tissue $\mathrm{pH}$ on the human fetus during labour using a new application technique. $\mathrm{Br} J$ Obstet Gynaecol 87 (1980) 666

(13) Lumley $J$ et al: The unreliability of a single estimation of fetal scalp blood pH. J Lab Clin Med 77 (1971) 535

(14) Renou P, C Wood, J Lumley: Intrapartum fetal monitoring. In: Laurensen NH (ed.) "Modern Management of High Risk Pregnancy". Plenum, New York 1983

(15) Suidan JS, BK Young, FW Hetzel et al: pH measurement with a fiberoptic tissue-pH monitor and a standard blood-pH meter. Clinical Chemistry 29 (1983) 1566

(16) Roby PV, HM Hochberg, HE Fox et al: $\mathrm{OBpH} 1000$ fetal tissue $\mathrm{pH}$ monitor clinical trial, Abst AAMI, Los Angeles 1987 